\title{
Electrocardiographic Alternans in Myocardial Bridge: A Case Report
}

\author{
Ilaria Marcantoni ${ }^{1}$, Alessia Di Menna ${ }^{1}$, Francesca Rossini ${ }^{1}$, Federica Turco ${ }^{1}$, Micaela Morettini ${ }^{1}$, \\ Agnese Sbrollini ${ }^{1}$, Francesco Bianco ${ }^{2}$, Marco Pozzi ${ }^{2}$, Laura Burattini ${ }^{1}$ \\ ${ }^{1}$ Università Politecnica delle Marche, Ancona, Italy \\ ${ }^{2}$ Ospedali Riuniti, Ancona, Italy
}

\begin{abstract}
Myocardial bridge $(M B)$ is a congenital heart condition in which a "bridge" of myocardium is overlying a "tunneled" coronary artery. $M B$ can be associated with a series of critical cardiac events. Aim of this study was to evaluate electrocardiographic alternans (ECGA) on a MB patient, being ECGA a cardiac electrical risk index defined as beat-to-beat alternation of electrocardiographic P-wave, QRS-complex and Twave morphology at stable heart rate. ECGA analysis was performed in a 1-hour 12-lead electrocardiographic recording of a 54 years-old $M B$ male patient at rest by application of the heart-rate adaptive match filter method. Areas of P-wave, QRS and T-wave alternans (PWAA, QRSAA, TWAA) were measured, evaluating also the prevalent among the three. Results showed the prevalent alternans was $T$-wave alternans, being TWAA on average equal to $6.3 \mu V \times s \quad(P W A A=4.7 \mu V \times s$, $Q R S A A=4.3 \mu V \times s)$; TWAA prevalence occurrence rate was 94\% (PWAA: 5\%, QRSAA:1\%). TWAA was also found to be significantly correlated $\left(\rho=0.72, p<10^{-2}\right)$ with heart rate. Eventually, TWAA was at least twice higher than in previously analyzed male healthy subjects. Thus, $M B$ seems to be associated to a higher cardiac electrical risk, possibly especially while performing physical activity at high heart rate.
\end{abstract}

\section{Introduction}

Myocardial bridging (MB) is a congenital heart anomaly consisting of an epicardial coronary artery that assumes an intramuscular course. Consequently, myocardium acts as a "bridge", since it overlies the coronary artery, so-called "tunneled artery", instead of being physiologically underneath it [1]. MB can affect the growth of the adjacent blood vessels, depending on its entity and depth [2]. Additionally, MB often affects the left anterior descending coronary, which results distorted during systole causing a delay in diastolic relaxation. This functional disturbance is usually relieved through surgical resection of the muscle bridge [3].
Despite some individuals are symptom free, MB is not always a benign finding and can be associated with cardiovascular complications [1]. Mechanical stress in correspondence of MB segment can cause endothelial damage, atherosclerosis and vasospasm, phenomena potentially leading to ischemia. Other complications of MB include also angina, acute coronary syndromes, left ventricular dysfunction and stunning, and arrhythmias [1,4-9]. Moreover, pathophysiological studies found a connection between the anomalous coronary blood flow resulting from MB and sudden cardiac death [4]. MB can cause myocardial fibrosis and edema, and both conditions lead to a higher risk of electrical instability. This happens especially in case of hemodynamically significant $\mathrm{MB}$, of which association with sudden cardiac death seems to be justified by an increased heart electrical heterogeneity [6].

The most powerful weapon against severe cardiac events, possibly associated with MB, remains prevention. Prevention can be supported using risk markers during standard clinical tests, such as the common electrocardiogram (ECG). An ECG risk marker for identification of pathologies linked to a higher risk of arrhythmias, or sudden cardiac death in the worst cases, is T-wave alternans (TWA) [10-12]. TWA is an electrophysiological phenomenon that consists of a beatto-beat fluctuation in the morphology of the $\mathrm{T}$ wave, including its amplitude, shape, and polarity. TWA is a risk index particularly for ventricular arrhythmias. However, a complete vision of possible electrical instability affecting any part of the heart and any phase of the cardiac cycle, thus reflecting into P-wave alternans (PWA) and/or QRS alternans (QRSA), could be very useful [13,14]. Therefore, ECG alternans (ECGA), defined as the prevalent alternans affecting ECG [15], should be considered. Among all computerized methods specifically designed for automatic TWA detection [1617] there is the adaptive match filter (AMF) method [18]. Recently, it was adapted also for PWA and QRSA detection, to achieve a comprehensive investigation of ECGA [15]. This study aims to evaluate ECGA at rest on a patient with $\mathrm{MB}$ by using the AMF in order to contribute to the knowledge of the relationship of MB 
with the predisposition to develop electrical cardiac anomalies.

\section{Data and Methods}

\subsection{Clinical Data}

The subject under examination is a male patient affected by MB, 54 years old and hospitalized before surgery at "Ospedali Riuniti" in Ancona. The coronary artery affected by $\mathrm{MB}$ is the left one, particularly the anterior descending branch. The patient underwent a 12lead-ECG recording, continuously acquired for 18 hours starting from 4:20 pm. The ECG was recorded through a wearable 12-lead M12 Holter ECG recorder by Global Instrumentation $® \quad$ (sampling rate: $1000 \mathrm{~Hz}$; www.globalinstrumentation.com;). During the acquisition the patient performed normal activities permitted by the hospitalization. Most of the time, but especially during the first hour, the patient was in a complete rest condition, lying on the bed.

The recording procedure was performed in accordance with the ethical principles of Helsinki Declaration and approved by the institutional expert committee and the patient gave his informed consent prior to acquisition.

\subsection{Automatic Electrocardiographic Alternans Detection}

All the available 12 ECG leads of the first hour of acquisition were analyzed for automatic ECGA detection through the AMF method [18]. Specifically, the 1-hour ECG tracing was splitted in consecutive and partially overlapping 64-beat windows with starting instants differing of $1 \mathrm{~s}$. Each ECG window underwent a preprocessing procedure according to which it was resampled from $1000 \mathrm{~Hz}$ to $200 \mathrm{~Hz}$ and filtered with a $6^{\text {th }}$ order bidirectional Butterworth bandpass filter, implemented as a cascade of a high pass filter with a cutoff frequency of $0.3 \mathrm{~Hz}$ and a low pass filter with a cutoff frequency of $35 \mathrm{~Hz}$. Identification of $\mathrm{R}$ peaks was performed always on one lead only. Since presence of heart-rate variability and/or ectopic beats can jeopardize alternans detection, each ECG window had to satisfy two suitability conditions, the first requiring standard deviation of RR intervals to be lower than $10 \%$ of mean $\mathrm{RR}$ interval, and the second requiring number of ectopic or noisy beats to be less than $10 \%$ of the total beats in the window. Ectopic and noisy beats were identified has those having a correlation coefficient with the median beat (computed over the beats of the window) less than 0.8 ; once identified, they were replaced with the median beat.

ECG windows not satisfying both suitability conditions were rejected; differently, they were accepted and submitted to the AMF for ECGA detection. ECGA was deemed to be characterized by a very narrow frequency band. Therefore, the AMF method was implemented as a $6^{\text {th }}$ order bidirectional Butterworth band-pass filter with cut-off frequencies at $1 /(2 \cdot$ mean $\mathrm{RR}) \pm 0.06 \mathrm{~Hz}$ [18]. The filtered ECG window is a pseudosinusoid the amplitude of which is zero if ECGA is not present or greater than zero otherwise. PWA, QRSA and TWA amplitudes $(\mu \mathrm{V})$ were computed as twice the pseudosinusoid amplitude in correspondence of the $\mathrm{P}$ wave, the QRS complex and the $\mathrm{T}$ wave, respectively [15]. Eventually, PWA, QRSA and TWA areas (PWAA, QRSAA, TWAA; $\mu \mathrm{V} \times \mathrm{s}$ ) were computed as the product of PWA amplitude and P-wave width, of QRSA amplitude and QRS width, and of TWA amplitude and T-wave width, respectively. Prevalent alternans was defined as the one with highest area. Eventually, the leads for which the number of rejected windows (rW) was less than $30 \%$ were considered reliable.

\subsection{Statistics}

Normality of PWAA, QRSAA and TWAA distributions relative to a single lead over the ECG windows were tested through the Lilliefors test. Normal and non-normal distributions were quantified in terms of mean \pm standard deviation and $50^{\text {th }}\left[25^{\text {th }} ; 75^{\text {th }}\right]$ percentiles, respectively. Mean values over reliable leads of the prevalent alternans area and of heart rate were computed every $5 \mathrm{~min}$ in order to obtain their trends over time. Finally, correlation between trends of prevalent alternans area against heart rate was tested. Statistical significance level $p$ was set at 0.05 in all cases.

\section{Results}

Analyzing the first hour of acquired 12-lead ECG and being the time step between consecutive windows equal to $1 \mathrm{~s}$, the number of preprocessed windows was 3600 for each lead.

Distributions of PWAA, QRSAA and TWAA are reported in Table 1 . Lead II was the one showing the highest alternans (PWAA=7.5 $\mu \mathrm{V} \times \mathrm{s}$, QRSAA=7.0 $\mu \mathrm{V} \times \mathrm{s}$ and TWAA $=10.0 \mu \mathrm{V} \times \mathrm{s})$. For each lead, the prevalent alternans was TWA, being its median TWAA greater than both median PWAA and median QRSAA. Such finding was also confirmed by averaging over the leads for which PWAA was $4.7 \mu \mathrm{V} \times \mathrm{s}$, QRSAA was $4.3 \mu \mathrm{V} \times \mathrm{s}$ and TWAA was $6.3 \mu \mathrm{V} \times \mathrm{s}$.

Results on prevalence occurrence rate are reported in Table 2. Occurrence rate of $\mathrm{rW}$ ranged from $19 \%$ to $50 \%$. Out of 12 leads, 6 were considered reliable (II, III, V1, V4, aVL, aVF); among these there is lead II which is the one showing the highest alternans. TWA confirmed to be the prevalent alternans. Indeed, TWA had a mean 
prevalence occurrence rate over all leads of 94\%, followed by PWA (5\%) and QRSA (1\%). Thus, TWA was the prevalent, even if not the only one present.

Trend of mean \pm standard deviation TWAA (i.e. the prevalent alternans) over reliable leads II, III, V1, V4, $\mathrm{aVL}$, and aVF is reported in Figure 1, where it is also depicted the trend of heart rate. Common trend shown by Figure 1 was confirmed by correlation between mean TWAA over reliable leads and heart rate: correlation was strong (0.72) and statistically significant $\left(p<10^{-2}\right)$.

Table 1. PWAA, QRSAA and TWAA distributions, expressed as $50^{\text {th }}\left[25^{\text {th. }} ; 75^{\text {th }}\right]$ percentiles. In bold the prevalent alternans.

\begin{tabular}{|c|c|c|c|}
\hline \multirow{2}{*}{ Lead } & \multicolumn{3}{|c|}{ ECGA area $(\mu \mathrm{V} \times s)$} \\
\hline & PWAA & QRSAA & TWAA \\
\hline I & $5.6[4.1 ; 7.7]$ & $5.4[4$ & $7.2[5.6 ; 9.4]$ \\
\hline II & 7.5 & 7.0 & 10.0 \\
\hline III & $4.7[3.7 ; 5.7]$ & $4.1[3.2 ; 5.1]$ & $6.6[5$. \\
\hline V1 & $6.4[4.5 ; 8.3]$ & $5.9[4.4 ; 7.8]$ & $8.2[6.6 ; 10.4]$ \\
\hline V2 & $3.7[2$. & $3.5[2$ & $5.2[4$ \\
\hline V3 & 4.8 & 4.2 & 0.6 \\
\hline V4 & & & 5.0 \\
\hline $\mathrm{Vs}$ & 2.8 & & 3.6 \\
\hline V6 & 4.5[ & 4.0 & 5.8[ \\
\hline aVR & $3.6[2$ & $3.3[2$ & $5.4[3.8$ \\
\hline aVL & $4.5[3.5 ; 6.4]$ & $4.1[3.0 ; 5.6]$ & $6.0[4.6 ; 8.4]$ \\
\hline aVF & $4.7[3.3 ; 7.0]$ & $4.3[3.1 ; 6.5]$ & $6.2[4.4 ; 9.8]$ \\
\hline
\end{tabular}

Table 2. Prevalence occurrence rate of PWA, QRSA, TWA and occurrence rate of rejected windows $(\mathrm{rW})$, expressed as percentage. In grey are highlighted the reliable leads $(\mathrm{rW}<30 \%)$.

\begin{tabular}{ccccc}
\hline \multirow{2}{*}{ Lead } & \multicolumn{3}{c}{$\begin{array}{c}\text { Prevalence occurrence } \\
\text { rate }(\%)\end{array}$} & $\begin{array}{c}\text { Occurrence } \\
\text { Rate }(\%)\end{array}$ \\
\cline { 2 - 4 } & PWA & QRSA & TWA & rW \\
\hline I & 6 & 1 & $\mathbf{5 7}$ & 36 \\
\hline II & 4 & 1 & $\mathbf{7 1}$ & 24 \\
III & 1 & 0 & $\mathbf{7 0}$ & 29 \\
V1 & 7 & 1 & $\mathbf{6 5}$ & 27 \\
\hline V2 & 1 & 0 & $\mathbf{4 9}$ & 50 \\
V3 & 1 & 0 & $\mathbf{5 0}$ & 49 \\
V4 & 6 & 1 & $\mathbf{7 1}$ & 22 \\
\hline V5 & 3 & 0 & $\mathbf{4 7}$ & 50 \\
V6 & 3 & 1 & $\mathbf{4 5}$ & 51 \\
aVR & 2 & 0 & $\mathbf{6 5}$ & 33 \\
\hline aVL & 3 & 0 & $\mathbf{7 7}$ & 20 \\
aVF & 2 & 0 & $\mathbf{7 9}$ & 19 \\
\hline
\end{tabular}

\section{Discussion}

This study evaluated ECGA at rest on a patient with MB by using the AMF in order to contribute to have more insight on the relationship of $\mathrm{MB}$ with an increased cardiovascular risk due to ECGA. The AMF method was chosen because of its ability to detect time variability of alternans and its versatility in detecting all possible types of alternans [15].

Since each ECG wave reflects a particular phase of the cardiac cycle, the entity of prevalent alternans is likely linked to the specific coronary artery affected by MB. In the case under examination, the patient had MB affecting a coronary that supplies blood to ventricles; thus, the anomaly was expected to most influence ventricular activity. Results indicate that the prevalent alternans was TWA, which overcame QRSA (also related to the electrical activity of ventricles). Eventually, PWA was smaller than TWA but not completely negligible, meaning an instability affecting all heart electrical activity, even if differently. Moreover, it is important to observe that MB seems to increase TWAA, and thus cardiac risk associated to it. Indeed, TWAA is, on average over $6 \mu \mathrm{V} \times \mathrm{s}$, with peaks over $10 \mu \mathrm{V} \times \mathrm{s}$, whereas TWAA measured on healthy male subjects by the same method is estimated to be around $3 \mu \mathrm{V} \times \mathrm{s}$ [19].

Thus, here TWAA is twice to three times higher than the expected one on a healthy male subject. Nothing can be said on PWA and QRSA since no reference values of PWAA and QRSAA in normal subjects are available in the literature.
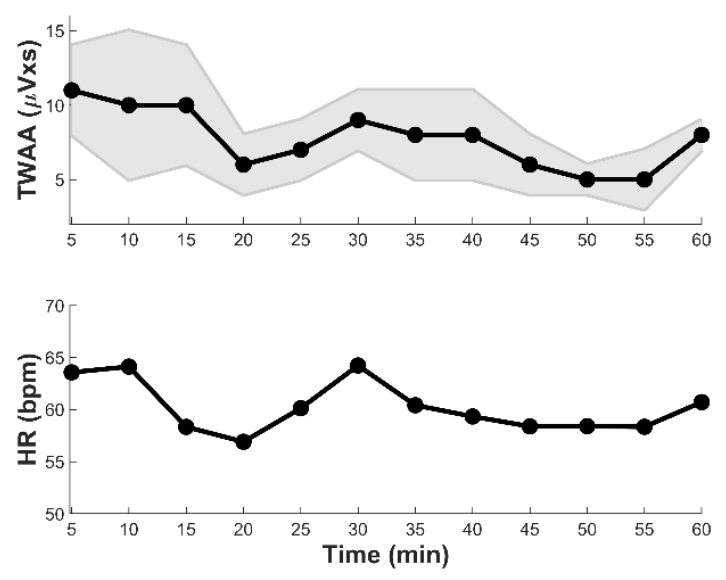

Figure 1. Trend of mean \pm standard deviation of TWAA over reliable leads (upper panel, where mean and standard deviation of TWAA are marked with full circles and through gray shade, respectively.) and heart rate (lower panel). 
Future studies will be aimed to determine physiological levels of PWA and QRSA in healthy subjects.

TWAA showed to have the typical behavior to be leaddependent (wide gray shade in Figure 1), as previously observed [20], and to correlate with heart rate. The dependence from heart rate could indicate that, in case of a subject affected by $\mathrm{MB}$, the risk to undergo arrhythmias, also severe, is not only higher than in healthy people, but it could even grow up while the subject is performing physical activity involving accelerated heart rate. Further studies are needed to confirm this hypothesis.

In conclusion, ECGA is higher than normal in our MB patient and manifests itself as TWA. Thus, in general, MB could be associated to an increased cardiac electrical risk, especially at high heart rate.

\section{References}

[1] M. S. Lee, and C. H. Chen, "Myocardial bridging: An upto-date review," J. Invasive Cardiol., vol. 27, no. 11, pp. 521-528, May 2015.

[2] A. G. Ferreira, S. E. Trotter, B. König, L. V. Decourt, K. Fox, and E. G. J. Olsen, "Myocardial bridges: Morphological and functional aspects," Br. Heart J., vol. 66, no. 5, pp. 364-367, Nov. 1991.

[3] R. C. Hill, W. R. Chitwood Jr, T. M. Bashore, J. D. Sink, J. L. Cox, and A. S. Wechsler, "Coronary flow and regional function before and after supraarterial myotomy for myocardial bridging," Ann. Thorac. Surg., vol. 31, no. 2, pp. 176-181, Feb. 1981.

[4] S. M. Yuan, "Myocardial bridging," Braz. J. Cardiovasc. Surg., vol. 31, no. 1, pp. 60-62, Jan.-Feb. 2016.

[5] A. R. Morales, R. Romanelli, and R. J. Boucek, "The mural left anterior descending coronary artery, strenuous exercise and sudden death," Circulation., vol. 62, no. 2, pp. 230237, Aug. 1980.

[6] S. Hostiuc, G. C. Curca, D. Dermengiu, S. Dermengiu, M. Hostiuc, and M. C. Rusu, "Morphological changes associated with hemodynamically significant myocardial bridges in sudden cardiac death," Thorac. Cardiovasc. Surg., vol. 59, no. 7, pp. 393-398, Oct. 2011.

[7] M. Agirbasli, G. S. Martin, J. Bunker Stout, H. S. Jennings, J. W. Lea, and J. H. Dixon, "Myocardial bridge as a cause of thrombus formation and myocardial infarction in a young athlete," Clin. Cardiol., vol. 20, no. 12, pp. 10321036, Dec. 1997.

[8] S. Kastellanos, K. Aznaouridis, C. Vlachopoulos, E. Tsiamis, E. Oikonomou, and D. Tousoulis, "Overview of coronary artery variants, aberrations and anomalies," World J. Cardiol., vol. 10, no. 10, pp. 127-140, Oct. 2018.
[9] Y. Ishikawa, Y. Kawawa, E. Kohda, K. Shimada, and T. Ishii, "Significance of the anatomical properties of a myocardial bridge in coronary heart disease," Circ. J., vol. 75, no. 7, pp. 1559-1566, Jul. 2011.

[10] I. Marcantoni, V. Cerquetti, V. Cotechini, M. Lattanzi, A. Sbrollini, M. Morettini, and L. Burattini, "T-wave alternans in partial epileptic patients," $45^{\text {th }}$ Comput. Cardiol. Conf., vol. 2018-September, Sep. 2018, Art. no. 8743701.

[11] C. Leoni, I. Marcantoni, A. Sbrollini, M. Morettini, and L. Burattini, "TWA Identifier for cardiac risk self-monitoring during hemodialysis: A case report," $23^{\text {rd }}$ IEEE ISCT 2019, pp. 143-146, Jun. 2019, Art. No. 8901032.

[12] L. Burattini, S. Bini, and R. Burattini, "Repolarization alternans heterogeneity in healthy subjects and acute myocardial infarction patients," Med. Eng. Phys., vol. 34, no. 3, pp. 305-312, Apr. 2012.

[13] P. Maury, and J. Metzger, "Alternans in QRS amplitude during ventricular tachycardia," Pacing Clin. Electrophysiol, vol. 25, no. 2, pp. 142-150, Feb. 2002.

[14] E. Siniorakis, S. Arvanitakis, P. Tzevelekos, N. Giannakopoulos, and S. Limberi, "P-wave alternans predicting imminent atrial flutter," Cardiol. J., vol.24, no. 6, pp.706-707, 2017.

[15] I. Marcantoni, D. Calabrese, G. Chiriatti, R. Melchionda, B. Pambianco, G. Rafaiani, E. Scardecchia, A. Sbrollini, M. Morettini, and L. Burattini, "Electrocardiographic alternans: A new approach," $15^{\text {th }}$ MEDICON 2019, IFMBE Proc., vol. 76, pp. 159-166, 2020.

[16]L. Burattini, S. Bini, R. Burattini, "Comparative analysis of methods for automatic detection and quantification of microvolt T-wave alternans," Med. Eng. Phys., vol. 31, no. 10, pp. 12901298, Dec. 2009.

[17] L. Burattini, S. Bini, R. Burattini, "Correlation method versus enhanced modified moving average method for automatic detection of T-wave alternans," Comput. Meth. Prog. Bio.,vol. 98, no. 1, pp- 94102, Apr. 2010.

[18] L. Burattini, W. Zareba, and R. Burattini, "Adaptive match filter based method for time vs. amplitude characterization of microvolt ECG T-wave alternans," Ann. Biomed. Eng., vol. 36, no. 9, pp. 1558-1564, Sep. 2008.

[19] L. Burattini, W. Zareba, and R. Burattini, "Identification of gender-related normality regions for T-wave alternans," Ann. Noninvasive Electrocardiol., vol. 15, no. 4, pp. 328336, Oct. 2010.

[20] L. Burattini, S. Man, R. Burattini, and C.A. Swenne, "Comparison of standard vs. orthogonal ECG leads for Twave alternans identification," Ann. Noninvasive Electrocardiol., vol. 17, no. 2; pp. 130-140, Apr. 2012.

Address for correspondence.

Laura Burattini.

Università Politecnica delle Marche,

Department of Information Engineering,

Via Brecce Bianche, 60131 Ancona, Italy.

E-mail address.1.burattini@univpm.it. 